\title{
Fatty liver (liver steatosis)
}

EASL-EASD-EASO

\section{Source}

. (2016). EASL-EASD-EASO Clinical Practice Guidelines for the management of non-

alcoholic fatty liver disease Journal of Hepatology, vol. 64 (6), 1388-1402.

doi:10.1016/j.jhep.2015.11.004

An hepatic triglyceride content $>5 \%$ as detected by liver biopsy. 